Several therapeutic sequences were applied in our study series, the most frequent was surgery associated with radiotherapy and chemotherapy (60.1\%). The direct annual cost of treatment was estimated at 1268502 DT $(\sim 465000$ \$). Radiotherapy represented the largest item of expenditure $(37.4 \%$ of the cost of treatment).

Conclusions The control infectious origin's pathology necessarily involves the implementation of national screening program, but also public awareness campaigns and mass vaccination against HPV of young virgin girls.

\section{EPV075/\#455 INTRAOPERATIVE LYMPH NODE FROZEN SECTION EXAMINATION (FSE) IN EARLY STAGE CERVICAL CANCER - A RISK STRATIFICATION ALGORITHM}

${ }^{1} S$ Smyth*, ${ }^{1} \mathrm{E}$ Jackson, ${ }^{2} \mathrm{~N}$ Sadeghi, ${ }^{3} \mathrm{C}$ Pinelli, ${ }^{1} \mathrm{~N}$ Ghanbarzadeh, ${ }^{1} \mathrm{C}$ Pappa, ${ }^{1} \mathrm{H}$ Jiang, ${ }^{4} \mathrm{Z}$ Traill, ${ }^{5} \mathrm{~S}$ Dhar, ${ }^{1} \mathrm{H}$ Soleymani Majd, ${ }^{1} \mathrm{M}$ Alazzam. ${ }^{1}$ Oxford University Hospitals NHS Foundation Trust, Gynaecological Oncology, Oxford, UK; ${ }^{2}$ Oxford University Hospitals NHS Foundation Trust, Obstetrics and Gynaecology, Oxford, UK; ${ }^{3}$ Del Ponte Hospital, University of Insubria, Obstetrics and Gynaecology, Varese, Italy; ${ }^{4}$ Oxford University Hospitals NHS Foundation Trust, Radiology, Oxford, UK; ${ }^{5}$ Oxford University Hospitals NHS Foundation Trust, Histopathology, Oxford, UK

\subsection{6/ijgc-2021-IGCS.143}

Objectives To evaluate pre-operative radiology and histopathology findings in cervical cancer lymphadenopathy detection, allowing targeted FSE.

Methods A retrospective analysis was conducted of 203 early stage cervical cancer patients between 2010 and 2019 in a tertiary centre. All patients had histologically confirmed cervical cancer and underwent MRI prior to intraoperative FSE.

Results 19 patients were found to have lymph node metastases (LNM) (9.36\%) at FSE. Patients were at increased risk of LNM by 6-fold with positive LVSI, 3-fold with MRI lymphadenopathy and 3.5-fold with MRI visible disease. The presence of lymphadenopathy on MRI and positive LVSI in combination increased the risk of LNM by 19 -fold.

Abstract EPV075/\#455 Table 1 Pre-operative risk stratification algorithm

Pre-operative risk stratification algorithm

\begin{tabular}{|c|c|c|c|}
\hline & Odds Ratio & $95 \% \mathrm{Cl}$ & Pvalue \\
\hline LVSI positive & 6.25 & $1.25-31.12$ & 0.02 \\
\hline MRI lymphadenopathy & 2.94 & $1.02-8.43$ & 0.04 \\
\hline MRI visible disease & 3.51 & $1.12-10.99$ & 0.03 \\
\hline 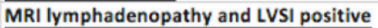 & 19.00 & $3.45-104.51$ & $0 . \overline{0007}$ \\
\hline
\end{tabular}

Conclusions We believe that intraoperative FSE has a role in the surgical management of early cervical cancer. However, we acknowledge that it is expensive and unpredictably time intensive, exposing patients to increased surgery duration and associated risk. We also recognise that it may not be feasible for all patients. By application of the preoperative risk stratification algorithm we demonstrate that FSE can be a useful tool to reduce surgical morbidity and avoid ineffective radical surgery or multimodal treatment in a cost effective manner in high-risk patients.

\section{EPV076/\#456 BEAU BIDEN CANCER MOONSHOT PROGRESS REPORT ON ADVANCED CERVICAL CANCER: PILOT PROJECT ON DNA/RNA EXTRACTION FROM RECURRENT AND METASTATIC CARCINOMA SPECIMENS}

${ }^{1} \mathrm{~A}$ Hari ${ }^{*},{ }^{2} \mathrm{M}$ Sill, ${ }^{3} \mathrm{~B}$ Monk, ${ }^{4} \mathrm{M}$ Birrer, ${ }^{2} \mathrm{H}$ Lankes, ${ }^{2} \mathrm{~V}$ Filiaci, ${ }^{5} \mathrm{~N}$ Ramirez, ${ }^{6} \mathrm{~L}$ Wei, ${ }^{1} \mathrm{~K}$ Tewari. ${ }^{1}$ UC Irvine, Gynecologic Oncology, Orange, USA; ${ }^{2} N R G$, Oncology, Bethesda, USA; ${ }^{3}$ Arizona Oncology (US Oncology Network), Gynecologic Oncology, Obstetrics and Gynecology, Phoenix, USA; ${ }^{4}$ Rockefeller Cancer Institute, Gynecologic Oncology, Little Rock, Arkansas, USA; ${ }^{5}$ Nationwide Children's Hospital, Pathology, Columbus, Ohio, USA; ${ }^{6}$ Roswell Park Cancer Institute, Computational Biology, Buffalo, New. York, USA

\subsection{6/ijgc-2021-IGCS.144}

Objectives Genomic and downstream signaling data informing tumor angiogenesis, DNA repair, and immunologic tolerance are required to develop targeted therapy against cervical cancer. The Cervical Cancer Genome Atlas (TCGA) is derived primarily from pre-invasive and early-stage disease, with under-representation of recurrent/metastatic specimens. NRG/ GOG-0240 is the phase 3 randomized trial that demonstrated a survival benefit with anti-angiogenesis therapy. Patients enrolled on this study provided tumor samples for whole genome sequencing and whole exome sequencing (to be performed at the New York Genomic Center (NYGC)), as well as RNA-seq and microRNA-seq (University of North Carolina (UNC)), and bioinformatics modeling (Roswell Park Cancer Institute). To determine the feasibility of DNA/RNA extraction from these relatively small, formalin-fixed paraffin-embedded (FFPE) specimens, we conducted a pilot study.

Methods Following pathology review at the NRG Biospecimen Bank at Nationwide Children's Hospital, DNA/RNA were coextracted using established protocols. All samples were required to contain at least 50\% tumor content for somatic mutation detection.

Results Forty-four out of 107 FFPE samples (41\%) underwent successful extraction. 36 were sent in the pilot study including $27(75 \%)$ squamous-cell and 9 (25\%) adenocarcinomas. Prior to transfer to NYGC, most samples were noted to have high genomic quality number with few having lower than 10,000 base pairs. Two were flagged for low quality secondary to degradation. One out of 36 samples sent to UNC did not provide sufficient RNA. Five samples were high risk for low DV200 (RNA fragment sizes < 200 base pairs).

Conclusions DNA/RNA extraction can be performed using recurrent/metastatic cervical cancer FFPE specimens.

\section{EPV077/\#472 CLINICAL IMPLICATIONS OF COMPUTED TOMOGRAPHY-BASED, ARTIFICIAL INTELLIGENCE- DRIVEN SARCOPENIA AND BODY COMPOSITION CHANGE DURING PRIMARY TREATMENT IN EARLY CERVICAL CANCER}

SI Kim*, Q Han, M Lee, J-W Kim. Seoul National University College of Medicine, Department of Obstetrics and Gynecology, Seoul, Korea, Republic of

\subsection{6/ijgc-2021-IGCS. 145}

Objectives To investigate the impact of sarcopenia and body composition on survival outcomes in patients with early-stage cervical cancer. 